\title{
Platelet adhesiveness in young patients with ischaemic stroke
}

\author{
SUBHASH C. SHARMA, G. P. VIJAYAN, M. L. SURI, AND H. N. SETH \\ From the Army Hospital, Delhi Cant-110010, India
}

SUMMARY A significant increase in platelet adhesiveness was observed in 13 patients who developed ischaemic stroke at high altitude and in 43 patients who developed stroke at low altitude when compared with a similar group of normal healthy volunteers. Eighty-eight percent of the patients were below the age of 40 years. The role of platelet adhesiveness in the pathogenesis of cerebral schaemia in young patients is discussed.

Ischaemic cerebrovascular diseases constitute more than $70 \%$ of cases of stroke in the older age group (Aring and Merritt, 1935; Cohen, 1955; Dalal et al., 1968). Atherosclerosis of cerebral vessels both extracranial and intracranial accentuated by hypertension, diabetes mellitus, and hyperlipidaemia is considered to be responsible for thromboembolic episodes and consequent cerebral infarction (Silverstein and Doniger, 1963; Fisher et al., 1965; Lavy et al., 1973).

Various investigators have suggested that some of the untoward and frequently devastating events of stroke may be related to platelets. Elevated platelet adhesiveness in patients with thromboembolic diseases has been reported by some investigators, but denied by others (Moolten et al., 1963; Hume, 1966; Kirby and Martin, 1966; Bygdeman and Wells, 1969; Mason and Summerlin, 1972). A significant increase of platelet adhesiveness has been reported by Prakash et al. (1970) in cerebral venous thrombosis and by Bedi et al. (1974) in cerebral arterial thrombosis while Gaston et al. (1971) and Ettinger et al. (1969) failed to find any change. A review of the literature does not show any report on strokes at high altitude; this study assumes further significance because of the younger age group of our patients. Platelet adhesiveness was estimated in 56 consecutive cases of cerebral ischaemic episode and compared with that of 50 normal healthy males of a similar age group. The results are reported.

\section{Material and methods}

One hundred and six individuals comprising 56

Received for publication 8 December 1976 cases of ischaemic cerebrovascular episode (cerebral thromboembolism) and 50 normal subjects of comparable age constitute the material for the present study. They were divided into three groups as follows:

\section{Group $A$}

Thirteen patients developed stroke at high altitude after a variable period of stay ranging from 3 to 18 months. They had been stationed during this period at altitudes of $3000 \mathrm{~m}$ and above.

\section{Group B}

Forty-three patients developed the stroke on the plains. They had never been to altitudes higher than $1000 \mathrm{~m}$.

\section{Group C}

Fifty normal healthy volunteers had never been exposed to high altitude.

All were male subjects in the age group 18 to 45 years.

\section{SELECTION OF CASES}

The criteria for the selection of cases was based on the report of the Advisory Council for the National Institute of Neurological Diseases and Blindness (Report by an ad hoc committee, 1958). Cases of haemorrhagic stroke were excluded by examination of cerebrospinal fluid. None of the patients had clinical or laboratory evidence of hypertension, diabetes mellitus, syphilis or any cardiac abnormality. A routine blood count and urine analysis together with ECG, EEG, and radiographs of the skull, chest, and cervical spine were carried out in each patient. Angiographic studies of the appropriate 
vessels were done to detect occlusive vascular disease and to exclude conditions which may mimic cerebral ischaemic episodes such as space-occupying lesions, aneurysms, and arteriovenous malformations.

Platelet adhesiveness was carried out by the method of Eastham (1964) using adenosine diphosphate. Two millilitres of fasting blood was collected in siliconised syringes in the early morning and was added to $2.4 \mathrm{mg}$ of ethylenediamine-tetra-acetic acid (EDTA) and $2 \mathrm{mg}$ of heparin in separate polystyrene bottles and mixed in a Matbern mixer at $30 \mathrm{rev} / \mathrm{min}$. To the heparinised sample $0.04 \mathrm{ml}$ of ADP solution was added (from a stock solution of $5 \mathrm{mg}$ in $20 \mathrm{ml}$ saline) and the bottles were returned to the mixer. After exactly 30 minutes the ADP-treated sample was transferred to another polystyrene bottle containing $2.4 \mathrm{mg}$ EDTA. Samples were mixed for 30 minutes and platelet counts were performed as soon as possible but in all cases within an hour of collection. Counts were performed in duplicate using the improved Neubaur chamber and the results were averaged. The platelet adhesiveness was expressed as a percentage as follows:

Total EDTA sample count - heparinised sample ADP count/Total EDTA sample count $\times 100$

Keeping in mind that factors such as diet (Philp and Wright, 1965), physical exertion (Pegrum et al., 1967), and smoking (Ashby et al., 1965) affect platelet adhesiveness, the procedure was standardised as rigidly as was practicable. All subjects were admitted to hospital. Normal subjects (group C) were detained in wards for at least four days for stabilisation before the test. They were resting in bed most of the time. The subjects had fasted overnight and no smoking was permitted for three hours prior to venepuncture. No drug known to affect the platelet behaviour was administered. Patients were studied usually three to four weeks after the onset of an acute episode, first because most of them were received as transfer cases from the peripheral hospitals and, secondly, to eliminate any abnormalities resulting from thromboembolic phenomena. Statistical analysis was done by the Student's $t$ test.

\section{Results}

The total platelet counts in the three groups were essentially similar. Mean platelet adhesiveness in groups $\mathrm{A}, \mathrm{B}$, and $\mathrm{C}$ was $71.0 \%, 73.3 \%$, and $62.2 \%$ respectively. The results of statistical analysis are given in the Table. Platelet adhesiveness was significantly high ( $P<0.001)$ in group $A$ and $B$ as compared to group C (Table). No significant difference in the adhesiveness was noted between
Table Age distribution and platelet adhesiveness in young patients with ischaemic stroke

\begin{tabular}{|c|c|c|c|c|c|}
\hline \multirow[t]{2}{*}{ Group } & \multicolumn{3}{|l|}{ Age } & \multirow{2}{*}{$\begin{array}{l}\text { Platelet } \\
\text { adhesiveness } \\
(\%)\end{array}$} & P-value \\
\hline & $\begin{array}{l}\text { Mean } \\
\text { (range) }\end{array}$ & $\begin{array}{l}<40 \\
\text { years }\end{array}$ & $\begin{array}{l}>40 \\
\text { years }\end{array}$ & & $A \vee B A \vee C B \vee C$ \\
\hline $\begin{array}{l}A \\
n=13 \\
B \\
n=43 \\
C \\
n=50\end{array}$ & $\begin{array}{l}31 \cdot 9 \\
(21-45) \\
33 \cdot 6 \\
(24-45) \\
31 \cdot 1 \\
(18-45)\end{array}$ & $\begin{array}{l}92 \% \\
86 \% \\
96 \%\end{array}$ & $\begin{array}{l}8 \% \\
14 \% \\
4 \%\end{array}$ & $\begin{array}{l}71 \cdot 0 \pm 8 \cdot 9 \\
73 \cdot 3 \pm 8 \cdot 6 \\
62 \cdot 2 \pm 5 \cdot 3\end{array}$ & $<0.001$ \\
\hline
\end{tabular}

Values of platelet adhesiveness are mean $\pm S D$. $n$ is the number of subjects. $\mathbf{N S}=$ not significant.

patients who developed a stroke during their stay at high altitude (group A) and those who had the episode on the plains (group B). The age distribution of the subjects is given in the Table.

\section{Discussion}

Cerebral thrombosis, like thrombosis elsewhere, could be due to changes either in the vessel wall, in the local pattern of blood flow, or in the composition of the blood. One of the major constituents of the blood which plays an important role in coagulation is the platelet and it may well initiate the process of intravascular coagulation. In the formation of thrombus, platelets initially adhere to an area of vessel lining. Other platelets then aggregate about this nidus to form platelet thrombus. Increased platelet adhesiveness may prove damaging in many ways. Besides the obvious mechanical effects of platelet thrombus on microcirculation, endothelium at the site of lodgement of platelet aggregate is injured by vasoactive substances released from them (Mustard, 1970). Bell et al. (1967) observed platelet emboli in cortical vessels during the development of experimental ischaemic infarction. Increased platelet aggregates have also been observed in patients with transient ischaemic attacks (Wu and Hoak, 1975).

Our data show that platelet adhesiveness is significantly increased in ischaemic stroke $(P<0.001)$ as compared to normal subjects. The majority ( $88 \%)$ of the patients were below 40 years of age. Danta (1970) and Acheson et al. (1972) have reported that there is no correlation between platelet count and platelet adhesiveness. They have also shown that the time gap between the onset of stroke and the performance of tests does not affect the platelet adhesiveness. Danta (1970) also came to the conclusion that platelet adhesiveness in cases of stroke does not change significantly during the next six weeks. In view of this it was considered that platelet adhesiveness as estimated after three to four weeks of the 
ischaemic episode would reflect a stabilised state. Our limited experience, based on a small number of patients who were admitted directly to this centre after the acute episode and were not put on any chemotherapeutic agents likely to affect the platelet functions, shows no significant change in platelet adhesiveness from the second to the ninth week (unpublished) and is in partial agreement with the above reports. Long-term, well-controlled serial studies in a large number of patients are, however, required to delineate clearly the pattern of platelet function in the post-stroke period. In the present study, owing to administrative reasons, it was not possible serially to estimate platelet adhesiveness in all the subjects. Only values obtained during a three to four week period were available for all the patients and these were analysed for the present study.

The onset of ischaemic stroke in these younger individuals in whom predisposing factors such as diabetes mellitus, hypertension, and ischaemic and valvular heart disease have been excluded suggest that significant atherosclerotic lesions in the extracranial vessels is unlikely to be a major contributory factor. That some of these patients may have been people with premature generalised atherosclerosis was kept in mind. Our observations on angiography in these patients favour the nonatherosclerotic origin of stroke in them. Increased platelet adhesiveness detected in groups A and B may have been responsible for the precipitation of cerebral ischaemic episodes in our patients.

A significant increase in platelet adhesiveness has been noted in normal healthy individuals exposed to high altitude (personal observations). Our data suggest that there is no statistically significant difference in platelet adhesiveness in those who developed stroke at high altitude (group A) compared with those who were affected on the plains (group B). But a higher percentage of patients in the younger age group were affected at high altitude (Table). It is likely that susceptible individuals may suffer from stroke at a younger age at high altitude. Long-term studies may be helpful to establish a direct relation between high-altitude exposure and the risk of stroke in young individuals. Our studies further strengthen the concept of management of ischaemic stroke, both preventive and therapeutic, by the use of antiplatelet adhesive agents (O'Brien, 1964; Wu and Hoak, 1975; Meyer, 1975).

We thank the Director General, Armed Forces Medical Services for permission to publish this paper.

\section{References}

Acheson, J., Danta, G., and Hutchinson, E. C. (1972). Platelet adhesiveness in patients with cerebral vascular disease. Atherosclerosis, 15, 123-127.

Aring, C. D. and Merritt, H. H. (1935). Differential diagnosis between cerebral hemorrhage and cerebral thrombosis. Archives of Internal Medicine, 56, 435-456.

Ashby, P., Dalby, A. M., and Millar, J. H. D. (1965). Smoking and platelet stickiness. Lancet, 2, 158-159.

Bedi, H. K., Bomb, B. S., Devpura, J. C., Vyas, B. R., and Bedi, T. (1974). Platelet adhesiveness in cerebral thrombosis. Journal of the Association of Physicians of India, 22, 829-831.

Bell, W. H., Sundt, T. M., Jr., and Nofzinger, J. D. (1967). The response of cortical vessels to serotinin in experimental cerebral infarction. Journal of Neurosurgery, 26, 203-212.

Bygdeman, S. and Wells, R. (1969). Studies of platelet adhesiveness, blood viscosity and the microcirculation in patients with thrombotic disease. Journal of Atherosclerosis Research, 10, 33-39.

Cohen, M. M. (1955). Cerebrovascular accidents. A study of two hundred and one cases. Archives of Pathology, 60, 296-307.

Dalal, P. M., Shah, P. M.. Aiyar, R. R., and Kikani, B. J. (1968). Cerebrovascular diseases in West Central India. British Medical Journal, 3, 769-774.

Danta, G. (1970). Platelet adhesiveness in cerebrovascular disease. Atherosclerosis, 11, 223-233.

Eastham, R. D. (1964). Rapid adhesive platelet count in whole blood. Journal of Clinical Pathology, 17, 45-46.

Ettinger, M. G., Kusonoki, R., and Fujishima, H. (1969). Blood coagulation studies. Geriatrics, 24 (10), 116-125.

Fisher, C. M., Gore, I., Okabe, N., and White, P. D. (1965). Atherosclerosis of carotid and vertebral arteries-extracranial and intracranial. Journal of Neuropathology and Experimental Neurology, 24, 455-476.

Gaston, L. W., Brooks, J. E., Blumenthal, H. J., and Miller, C. E. (1971). A study of blood coagulation following an acute stroke. Stroke, 2, 81-87.

Hume, M. (1966). Platelet adhesiveness and other coagulation factors in thrombophlebitis. Surgery, 59, 110-116.

Kirby, J. C., Jr. and Martin, C. L. (1966). Platelet adhesiveness and vascular disease. Circulation, 34, 17 (Supplement III).

Lavy, S., Melamed, E., Cahane, E., and Carmon, A. (1973). Hypertension and diabetes as risk factors in stroke patients. Stroke, 4, 751-759.

Mason, R. G. and Summerlin, D. C. (1972). Alteration of platelet adhesion to glass in vascular disorders and certain other diseases. American Journal of Clinical Pathology, 57, 611-617.

Meyer, J. S. (1975). Summary of the international round table conference on platelet aggregation in the pathogenesis of cerebrovascular disorders. Stroke, 6, 239-244.

Moolten, S. E., Jennings, P. B., and Solden, A. (1963). Dietary fat and platelet adhesiveness in arteriosclerosis and diabetes. American Journal of Cardiology, 11, 290-300.

Mustard, J. F. (1970). Platelets in thromboembolic disease. Advances in Cardiology, 4, 131-142. 
O'Brien, J. R. (1964). The mechanism and prevention of platelet adhesion and aggregation considered in relation to arterial thrombosis. Blood, 24, 309-314.

Pegrum, G. D., Harrison, K. M., Shaw, S., Haselton, A., and Wolff, S. (1967). Effect of prolonged exercise on platelet adhesiveness. Nature, 213, 301-302.

Philp, R. B. and Wright, H. P. (1965). Effect of adenosine on platelet adhesiveness in fasting and lipaemic bloods. Lancet, 2, 208-209.

Prakash, C., Arya, R. K., Singla, K. P., and Bansal, B. C. (1970). Study of platelet adhesiveness and serum lipids in cerebral venous/venous sinus thrombosis during puerperium. Journal of the Association of Physicians of India, 18, 815-819.

Report by an ad hoc committee (1958). A classification and outline of cerebrovascular disease. Neurology (Minneapolis), 8, 397-434.

Silverstein, A. and Doniger, D. E. (1963). Systemic and local conditions predisposing to ischemic and occlusive cerebrovascular disease. Journal of the Mount Sinai Hospital, 30, 435-450.

Wu, K. K. and Hoak, J. C. (1975). Increased platelet aggregates in patients with transient ischemic attacks. Stroke, 6, 521-524. 